\section{Hipotireoidismo na gestação}

\section{Hypothyroidism in pregnancy}

\begin{abstract}
Pregnancy induces many physiological changes in maternal thyroid function. Furthermore, the presence of thyroid autoimmunity or iodine deficiency can exacerbate these alterations, resulting in maternal and/or fetal hypothyroidism and further occasional complications for the mother and the development of the fetus. Several studies have reported that untreated hypothyroidism during pregnancy may cause a significant decrease in intellectual development of the offspring. The aim of this review of the literature is to show the importance of identification and early therapy in pregnant women thus eliminating the risk of complications. We also point that women with previously diagnosed hipothyroidism should be advised to stabilize their disease before becoming pregnant to avoid these complications.
\end{abstract}

Key words Pregnancy, Hypothyroidism, Thyroid gland
Sheila Mamede da Costa 1

Lino Sieiro Netto 2

Alexandre Buescu 3

Mario Vaisman 4

\author{
1-4 Serviço de Endocrinologia . Faculdade de Medicina. \\ Hospital Universitário Clementino FragaFilho. \\ Universidade Federal do Rio de Janeiro. Sala FE23. \\ Av. Brigadeiro Tromposwiski, s.n. Ilha do Fundão, \\ Rio de Janeiro, RJ, Brasil. CEP: 21.941.590
}

Resumo A gestação induz mudanças fisiológicas na função tireoidiana materna. Além disso a presença de auto-imunidade tireoidiana ou de deficiência de iodo exacerbam essas alterações, podendo resultar em hipotireoidismo materno elou fetal e desta forma ocasionar complicações para as mães e o desenvolvimento dos fetos. Vários estudos têm demonstrado que filhos de mães com hipotireoidismo não tratado durante a gestação, podem apresentar comprometimento do desenvolvimento intelectual. O objetivo desta revisão bibliográfica é mostrar a importância de identificar e tratar precocemente as gestantes com essa enfermidade, e dessa forma eliminar os riscos de complicações. Recomenda-se também que as mulheres com diagnóstico prévio de hipotireoidismo devem ser aconselhadas a estabilizar a sua doença antes da gestação e assim previnirem em complicações.

Palavras-chave Gestação, Hipotireoidismo, Glândula tireóide 


\section{Introdução}

É comum o surgimento de desordens tireoidianas em mulheres na idade reprodutiva e durante a gestação.1 A freqüência de hipotireoidismo na gestação varia em cada país, porém estima-se em torno de $0,3 \%$ a $25 \%$. Nos países que não apresentam deficiência de iodo, a doença tireoidiana auto-imune é a principal causa de hipotireoidismo. A disfunção está relacionada a um grande número de complicações para a mãe e para o desenvolvimento dos fetos, sendo as mais freqüentes a hipertensão gestacional e o baixo peso fetal. 2

Vários estudos têm demonstrado que a presença de anticorpos anti-tireoidianos especialmente o anticorpo antiperoxidase tireodiana, pode ocasionar complicações para a mãe e o neonato, tais como, a deteriorização da função tireoidiana das mães e aumento de abortos espontâneos. Mulheres com abortos no primeiro trimestre de gestação apresentam níveis elevados de anti-TPO em comparação com aquelas que não o tiveram. ${ }^{3-5}$

$\mathrm{O}$ período gestacional representa um estresse para a glândula tireóide. Mulheres provenientes de regiões com deficiência moderada de iodo (ingestão $<100 \mu \mathrm{g} / \mathrm{dl}$ ) têm aumento da concentração de TSH durante a gestação, comparadas com mulheres de áreas suficientes de iodo. Esse aumento de TSH é maior naquelas que apresentam auto-imunidade tireoidiana, podendo levar a hipotireoidismo materno e/ou fetal.3,6 Vários estudos têm mostrado que o desenvolvimento intelectual dos filhos das mães com hipotireoidismo pode ser comprometido.2,3,7

$\mathrm{O}$ rastreamento das gestantes com risco de hipotireoidismo deve ser realizado rotineiramente, pois o tratamento com levotiroxina pode atenuar ou eliminar o risco de complicações. 8

\section{Função tireoidiana durante a gestação}

A gravidez é um período que induz algumas mudanças fisiológicas, modificando dessa forma a função tireoidiana. Três séries de eventos ocorrem em tempos diferentes, resultando em efeitos complexos que podem ser somente transitórios ou persistir até o termo.

A primeira seqüência de eventos começa durante a primeira metade da gestação e se mantém até o termo. Os hormônios tireoidianos são transportados no sangue através de três proteínas: globulina transportadora da tiroxina (TBG), transtirretina e albumina. Embora a TBG esteja presente em baixa concentração no nível sérico, apresenta alta afinidade com os hormônios tireoidianos sendo responsável por grande parte do transporte de T4 (68\%) e T3 (80\%). Durante a gestação, a afinidade das três proteínas por T4 e T3 não são significativamente alteradas, mas a concentração de TBG aumenta aproximadamente de duas a três vezes, enquanto que a concentração de albumina e transtirretina permanecem inalteradas. $\mathrm{O}$ nível sérico de TBG aumenta nas primeiras semanas da concepção, com pico em torno da $21^{\mathrm{a}}$ semana de gestação, mantendo-se assim até o final desse período. Os níveis elevados de estrógenos induzem esse aumento pelo efeito combinado de aumentar a síntese hepática e, também, a sialização da mólecula de TBG, levando desta forma a diminuição de seu clearance hepático em relação à mulher não grávida. Como resultado dessas alterações os níveis de T3 e T4 total aumentam durante a gestação, com tendência a situar-se no limite superior da normalidade. No início da gestação esses níveis costumam elevar-se acentuadamente e, mantêm um platô no início do segundo trimestre em cerca de $30-100 \%$ dos valores antes da gestação. A concentração dos hormônios livres tendem a reduzir seus valores, com um ligeiro aumento do TSH, resultante da estimulação do eixo hipófise-tireóide. Para a mulher grávida que reside em área suficiente de iodo, a diminuição na fração livre é limitada em aproximadamente 10 a $15 \%$, já em locais de restrição ou deficiência de iodo a redução é significativamente mais pronunciada. ${ }^{9-11}$

A segunda seqüência de eventos ocorre transitoriamente durante o primeiro trimestre e resulta da estimulação direta da tireóide materna por níveis elevados de gonadotrofina coriônica (hCG), que é acompanhada por uma inibição parcial do eixo hipófise-tireóide. Entre 8-14 semanas de gestação, há uma diminuição transitória no TSH sérico, coincidente com o pico na concentração de hCG.9,11 Em aproximadamente $20 \%$ das grávidas normais são observados níveis de TSH no limite inferior da normalidade $(<0,20 \mathrm{mU} / \mathrm{L})$, sendo os níveis de hCG significativamente maiores em comparação com $80 \%$ das grávidas que mantêm os níveis de TSH inalterados.10,11 A ação estimulatória do hCG pode de um modo geral, ser quantificada: um incremento de $10.000 \mathrm{IU} / \mathrm{L}$ está associado com um aumento médio de T4 livre de 0,6 pmol/L, e uma diminuição no TSH de $0,1 \mathrm{mU} / \mathrm{L}$. A fim de se tornar clinicamente aparente (induzir tireotoxicose gestacional), os níveis circulantes de hCG normalmente excedem 50.000-75.000 IU/L, permanecendo assim por um período prolongado, enquanto que na maioria das grávidas, o pico de hCG dura somente poucos dias. Consequentemente, na maior parte das gestantes normais, esse efeito estimulatório permanece 
secundário, e com curta duração, não sendo rotineiramente detectáve.1,9,12

A terceira seqüência de eventos inicia-se na segunda metade da gestação, sendo relacionada com modificações no metabolismo periférico dos hormônios tireoidianos maternos. Três enzimas cata-lizam a desiodação dos hormônios tireoideos nos tecidos humanos. A atividade da deiodase Tipo I provavelmente não é modificada durante a gravidez. A deiodase Tipo II é expressa na placenta e sua atividade pode representar um mecanismo homeostático para a manutenção da produção de T3 localmente, quando as concentrações de T4 estão reduzidas. A placenta também contém grandes quantidades de deiodase Tipo III, enzima que converte T4 para T3 reverso, e T3 para T2. Por sua atividade enzimática extremamente alta, durante a vida fetal, a deiodase Tipo III pode explicar o T3 baixo e concentrações de T3 reverso alta, que são característicos do metabolismo hormonal da tireóide fetal. 9,13

Em conjunto, alterações metabólicas associadas com a progressão da primeira metade da gestação constituem uma fase transitória de um estado de préconcepção para gravidez. Para que efetivamente ocorram, tais alterações requerem uma produção hormonal aumentada pela glândula tireóide materna. Uma vez que um novo equilíbrio tenha sido atingido, as demandas hormonais aumentadas são mantidas até o termo, provavelmente através da passagem transplacentária de hormônios da tireóide materna e aumento do turnover de T4 materno, presumivelmente sob a influência da alta atividade da deiodase Tipo III.

Para gestantes de países que não apresentem deficiência de iodo, o desafio da tireóide materna é ajustar a produção hormonal a fim de atingir um novo estado de equilíbrio, e depois disso, mantê-lo até o termo. Todavia essas adaptações fisiológicas são atingidas sem dificuldades pela tireóide normal. Contudo, esse não é o caso quando a capacidade funcional da glândula está prejudicada, como ocorre na doença tireoidiana auto-imune, hipotireoidismo ou quando a gestante reside em área deficiente de iodo. 9,14

\section{Hipotireoidismo materno}

A associação de hipotireoidismo com anovulação fez com que alguns médicos concluíssem que gestações complicadas por hipotireoidismo eram extremamente raras. 3,15 Posteriormente vários estudos demonstraram que essas mulheres podem engravidar, assim como apresentarem repercussões para elas e para o desen- volvimento dos seus fetos, caso a doença não seja adequadamente tratada. 3,16

As mudanças que ocorrem na função tireoidiana na gestação são sutis, quando a glândula e a ingesta de iodo são normais. Contudo o estresse da doença auto-imune e/ou a deficiência de iodo são suficientes para resultar em hipotireoidismo gestacional em algumas mulheres. 17

O sistema nervoso central fetal depende de iodo e tiroxina para o seu desenvolvimento durante toda a gestação, sendo dessa forma necessária uma avaliação das crianças nascidas de mães com severa deficiência de iodo. Para tentar determinar a importância do iodo na gestação, Cao et al.,18 demonstraram que das gestantes que receberam iodo durante o primeiro e segundo trimestres, somente $2 \%$ de seus filhos apresentaram moderada ou severa anormalidade neurológicas. Contudo, caso essa suplementação seja feita tardiamente durante o terceiro trimestre ou no início do pós-parto, as anormalidades aumentam para aproximadamente $9 \% .2,18$

O hipotireoidismo materno é comumente causado por tireoidite auto-imune ou por destruição da glândula (ablação com iodo ou cirurgia), e raramente por desordem hipotalâmica-hipofisária. A prevalência durante a gestação varia em cada país. ${ }^{2}$ Klein $e t$ al., 19 nos EUA ,encontraram aumento de TSH (>6 $\mathrm{mU} / \mathrm{L})$ em $49(2,5 \%)$ das 2000 gestantes avaliadas entre $15^{\mathrm{a}}$ a $18^{\mathrm{a}}$ semanas de gestação e diminuição do T4 em 0,3\%. Glinoer, 10 na Bélgica, analisou 1900 gestantes encontrando $2,2 \%$ de hipotireoidismo. ${ }^{11}$ No Japão, Fukushi et al., 20 observaram hipotireodismo em $102(0,14 \%)$ das 70.632 gestantes avaliadas.

Vários são os efeitos do hipotireoidismo não tratado para as mães e seus fetos (Quadro 1).2,21 Leung et al.,22 acompanharam 68 mulheres com hipotireoidismo durante toda a gestação, das quais 23 apresentavam hipotireoidismo clínico e 45 subclínico. Como resultado a prevalência de hipertensão gestacional foi elevada em ambos os grupos, sendo de $22 \%$ para o clínico e $15 \%$ no subclínico, em comparação com 7,6\% do grupo sem disfunção. Nos recém-nascidos, baixo peso foi a complicação mais comum dos filhos das mães com hipotireoidismo clínico, sendo encontrado em aproximadamente $22 \%$. Presença de malformações congênitas e natimortos foram observadas nas gestantes que não fizeram uso adequado do hormônio tireoidiano.22 Também com o objetivo de avaliar as conseqüências do hipotireoidismo na gestação, Davis et al.,23 acompanharam 16 mulheres com hipotireoidismo clínico, e dessas, sete apresentaram pré-eclâmpsia e três placenta prévia e hemorragia pós-parto. Nesse grupo foi observado baixo peso fetal $(<2000 \mathrm{~g})$ em cinco mulheres e dois nati- 
mortos. Das 12 gestantes com hipotireoidismo subclínico, duas apresentaram pré-eclâmpsia e hemorragia pós-parto. 23 Caso o hipotireoidismo clínico ou sub-clínico não seja tratado adequadamente, pode relacionar-se com o aumento do número de abortos espontâneos. Além disso, essas mulheres apresentam freqüentemente anti-TPO positivo ( $80 \%$ versus $9 \%$ das mulheres com TSH normal), e alguns estudos têm confirmado um aumento da incidência de abortos durante o primeiro trimestre de gestação.24 Vaquero et al. 25 demonstraram recentemente que a reposição de $\mathrm{T} 4$ poderia beneficiar um pequeno grupo dessas mulheres com história de abortos recorrentes.

Tem sido demonstrado em vários estudos que filhos de mães com hipotireoidismo descompensado durante a gestação podem apresentar significante decréscimo do quociente de inteligência (QI). Aproximadamente $20 \%$ dessas crianças tem níveis de QI igual ou menor 85, mostrando desta forma a importância da avaliação da função tireoidiana antes ou durante o início da gestação.2, , ,26

\section{Diagnóstico laboratorial}

O indicador mais sensível do hipotireoidismo é a determinação sérica do TSH, o qual se encontra invariavelmente elevado no hipotireoidismo primário, sobretudo em mulheres de regiões com deficiência de iodo. As concentrações de T4 livre podem estar dentro dos limites da normalidade em pacientes com hipotireoidismo subclínico, tendendo a diminuir de acordo com gravidade do quadro. Os níveis de T3 livre podem estar ainda normais mesmo quando o $\mathrm{T} 4$ livre já se encontra em níveis baixos. Os níveis de T3 e T4 totais, encontram-se elevados durante a gestação em função do aumento da concentração de TBG, não sendo utilizados para o diagnóstico. A determinação de anticorpos anti-TPO é importante na identificação de tireoidite de Hashimoto como a
Quadro 1

Hipotireoidismo materno: efeitos na mãe e feto.

\begin{tabular}{ll}
\hline Materno & Fetal \\
\hline Hipertensão Gestacional & $\begin{array}{l}\text { Prematuridade } \\
\text { Pré-eclâmpsia }\end{array}$ \\
Placenta prévia & Natimo peso ao nascimento \\
Anemia & Sofrimento fetal \\
Hemorragia pós-parto & \\
\hline
\end{tabular}

Adaptado de Smallridge RC. Hypothyroidism and pregnancy. Endocrinologist 2002; 12: 454-63.2 etiologia do hipotireoidismo, além de ser um marcador de risco das pacientes que podem desenvolver a doença durante a gravidez ou disfunção tireoidiana pós-parto. Tem sido relatada também a associação de anti-TPO a um risco aumentado de aborto espontâneo em aproximadamente $17 \%$ de gestantes, quando comparado com $8,4 \%$ em mulheres com anticorpos negativos. $1,2,27,28$

Portanto, o rastreamento durante a gestação (Figura 1) é justificado devido às complicações obstétricas e fetais associadas com hipotireoidismo não tratado, assim como a avaliação das pacientes com risco para desenvolvimento de disfunção tireoidiana pós-parto, especialmente tireoidite pós-parto (TPP).28 
Algorítmo de rastreamento para disfunção tireoidiana na gestação e pós-parto.

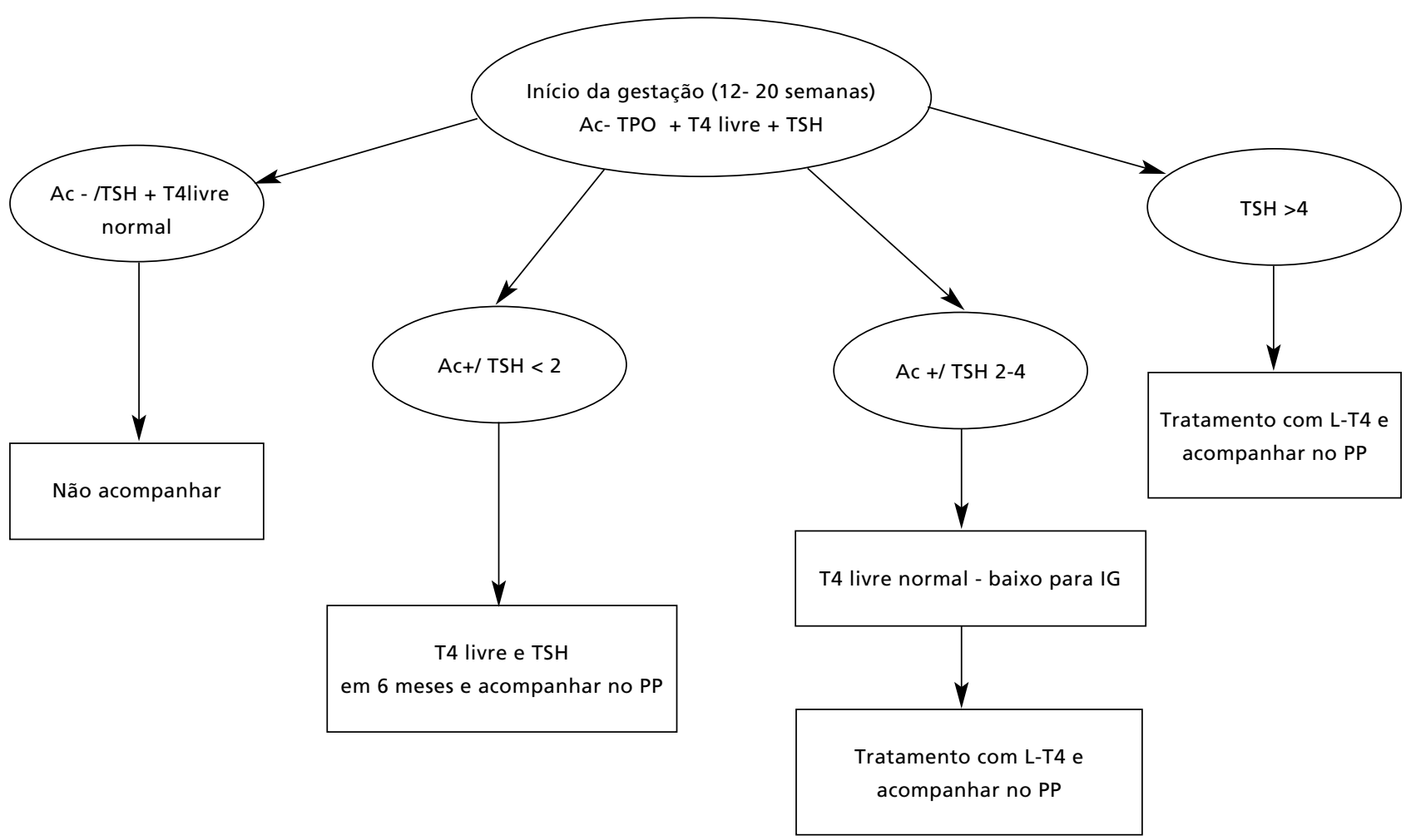

$\mathrm{AC}-\mathrm{TPO}=$ Anticorpo peroxidase tireoidiana; $\mathrm{AC}=$ Anticorpo negativo); $\mathrm{AC}+=$ Anticorpo positivo; IG = Idade gestacional; L-T4 = Levotiroxina; PP = Pós-parto

Fonte: Adaptado de Glinoer D. Management of hypo and hyperthyroidism during pregnancy. Growth Horm IGF Res 2003; 13: S45-S54. 28

\section{Tratamento}

Todas as mulheres portadoras de hipotireoidismo, especialmente aquelas com pretensão a engravidar, devem ser estimuladas a obter um bom controle da sua doença antes da concepção. As mulheres com diagnóstico e tratamento prévio à gestação devem ter o TSH e o T4 livre monitorados a partir do primeiro trimestre, com revisão a cada seis a oito semanas, devendo a dose da reposição com Levotiroxina (LT4) ser ajustada caso necessário. Tem sido observada, com muita freqüência por diversos autores, a necessidade de aumento da dose de reposição de T4 em aproximadamente 25 a $50 \%$ das doses utilizadas na pré-concepção, iniciando a partir do $1^{\circ}$ trimestre, e persistindo até o final da gestação. Essas necessidades aumentadas cessam no período de pós-parto, retornando à dose habitual pré-gestacional. Várias são as causas que tentam explicar a etiologia do aumento da necessidade de T4. No primeiro trimestre ocorre aumento do pool de TBG permanecendo assim até o final da gestação, e durante o segunda metade da gestação há aumento do tamanho da placenta, com maior atividade da deiodase tipo III inativando os hormônios tireoidianos, e, também, a expansão do volume plasmático. Tem sido sugerido que gestantes que fazem suplementação com sulfato de ferro e que reduz a absorção de $\mathrm{T} 4$, necessitam também de aumento da dose da reposição hormonal.3,29,30

Tem sido demonstrado que esse aumento da necessidade de Levotiroxina está relacionado com a etiologia do hipotireoidismo. Foi observado que as pacientes atireóticas por ablação cirúrgica e/ou radioiodo necessitaram aumentar a dose da reposição 
significativamente durante a gestação em comparação com as pacientes com hipotireoidismo por tireoidite de Hashimoto que necessitaram de um aumento muito menor, provavelmente pela presença de uma reserva tireóidea residual com capacidade de compensar as necessidades requeridas. 3,31

Nas pacientes em que o diagnóstico é realizado durante a gravidez o reajuste da dose deve ser feito com cerca de seis semanas após o início do tratamento, devendo elas ser avaliadas durante toda a gestação com a dosagem de TSH verificada a cada seis a oito semanas. As pacientes submetidas à ressecção de tireóide para tratamento de neoplasia e que se encontram em reposição de doses suprafisiológicas de hormônio como estratégia de tratamento (supressão) necessitam de aumento da dose já no primeiro trimestre de gestação.

Sabendo que a avaliação correta do hipotireoidismo antes e durante a gestação é fundamental para evitar as conseqüências para as mães e desenvolvimento dos fetos, Brent baseado em vários estudos, elaborou diretrizes para o manejo da doença (Quadro 2).3

Quadro 2

Diretrizes para avaliação de mulheres com hipotireoidismo antes e durante a gestação.

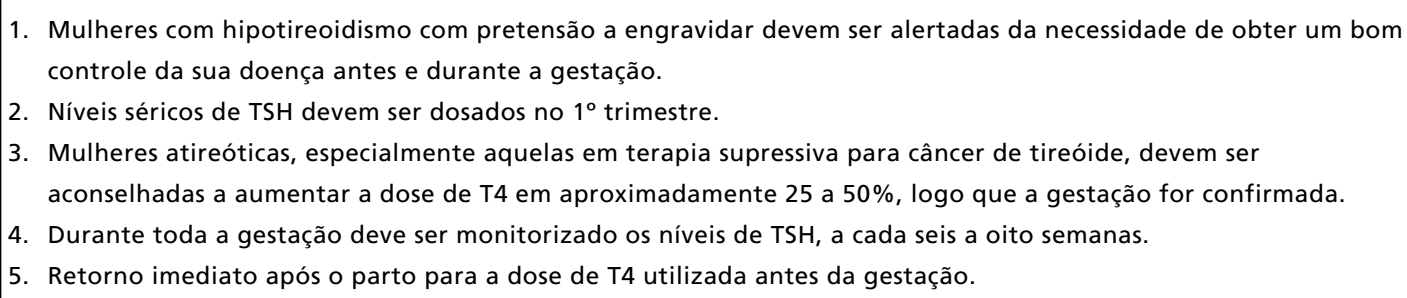

Fonte: Adaptado de Brent GA. Thyroid 1999; 9: 663. 3

\section{Disfunção tireoidiana pós-parto}

Embora esta revisão esteja direcionada para a gestação, é importante lembrar que o período de pósparto também é propício para o aparecimento de disfunção tireoidiana, sendo a causa mais comum TPP. $1,3,4$

Durante a gravidez, o sistema imune materno é suprimido, o que pode ocorrer devido a atividade aumentada das células $\mathrm{T}$ supressoras fetais. 32 No final da gravidez e durante os três primeiros meses de pós-parto ocorre uma queda das células T CD4 (células T helper) e um aumento das células T CD8 (células T supressoras), causando queda da relação CD4/CD8. Mulheres que desenvolvem TPP têm uma alta relação CD4/CD8 e ativação das células T CD4 no período de pós-parto. Após o parto o desaparecimento rápido dos fatores supressores de imunidade materna leva a um "rebote" na função auto-imune. Portanto os títulos de anticorpos tireoidianos apresentam tendência para a redução durante a gestação, alcançando um nadir no parto, com um pico no terceiro e quarto mês de pós-parto. 29,33

A TPP é uma disfunção causada pela destruição auto-imune da tireóide, acometendo aproximadamente 5 a $10 \%$ das mulheres no primeiro ano de pósparto, caracterizado por transitório hipertireoidismo hipotireoidismo ou ambos, sendo que aproximadamente 25 a $30 \%$ podem desenvolver hipotireoidismo permanente. As pacientes com TPP podem também apresentar recorrência em até $69 \%$ da doença em gestações subsequentes. 33,34

$\mathrm{O}$ anticorpo anti-TPO é considerado como o principal marcador da doença. Lazarus et al.,35 estudaram 152 gestantes com anti-TPO positivo (dosagem realizada até a $16^{\mathrm{a}}$ semana de gestação) e compararam com 239 mulheres com anticorpo negativo acompanhadas mensalmente durante os 12 meses de pós-parto, tendo observado TPP em aproximadamente $50 \%$ daquelas com auto-imunidade.

A maioria das pacientes com TPP tem manifestações clínicas leves de tireotoxicose e/ou hipoti- 
reoidismo, sendo freqüente que a doença passe despercebida por médicos e pacientes por associarem os sintomas ao período de pós-parto.

Laboratorialmente, as dosagens de T4 Livre e TSH variam de acordo com as diversas fases da disfunção tireóidea, sendo a captação de iodo 131 baixa na fase de tireotoxicose, fazendo, dessa forma, a diferenciação com a doença de Graves. 36
Diversos autores têm sugerido que todas as mulheres devam ser submetidas ao rastreamento para TPP durante o primeiro trimestre de gestação com a dosagem de anti-TPO, sendo aquelas com títulos elevados acompanhadas com T4 Livre e TSH durante os 12 meses de pós-parto, com a finalidade de identificar casos da doença. 34,37

\section{Referências}

1. Fantz RC, Dagogo-Jack S, Landenson HJ, Gronowski MA. Thyroid function during pregnancy. Clin Chem 1999; 45: 2250-8.

2. Smallridge RC. Hypothyroidism and pregnancy. Endocrinologist 2002; 12: 454-63.

3. Brent GA. Maternal hypothyroidism: recognition and management. Thyroid 1999; 9: 661-5.

4. Glinoer D, Fernandez-Soto ML, Bourdnoux P, Lejeune B, Delange F, Lemone M, Kinthaert J, Robijn C, Grun JP, De Nayer P. Pregnancy in patients with mild thyroid abnormalities: maternal and neonatal repercussions. J Clin Endocrinol Metab 1991: 73: 421-7.

5. Stagnaro-Green A, Roman SH, Cobin RH, EL-Harazy E, Alvarez-Marfany M, Davies TF. Detection of at risk pregnancy by means of highly sensitive assays for thyroid autoantibodies. JAMA 1990; 264: 1422-5.

6. Glinoer D, Delange F, Laboureur I, De Nayer PH. Maternal and neonatal thyroid function at birth in na area of marginally low iodine intake. J Clin Endocrinol Metab 1992; 75: 800-5.

7. Morreale de Escobar G, Obregon MJ, Escobar del Rey F. Is neuropsychological development related to maternal hypothyroidism or to maternal hypothyroxinemia? J Clin Endocrinol Metab 2000; 85: 3975-87.

8. Mandel SJ, Brent GA, Larsen PR. Levothyroxine therapy in patients with thyroid disease. Ann Intern Med 1993; 119: 492-502.

9. Glinoer D. What happens to the normal thyroid during pregnancy? Thyroid 1999; 9: 631-5.

10. Glinoer $\mathrm{D}$. The regulation of thyroid function in pregnancy pathways of endocrine adaptation from physiology to pathology. Endocrinol Rev 1997; 18: 404-3.

11. Glinoer D, De Nayer P, Bourdoux P, Lemone M, Robyn C, Van Steirteghem A, Kinthaert J, Lejeune B. Regulation of maternal thyroid during pregnancy. J Clin Endocrinol Metab 1990;71: 276-87.

12. Grun JP, Meuris S, De Nayer P, Glinoer D. The thyrotropic role of human chorionic gonadotropin (hCG) in the early stages of twin (versus single) pregnancy. Clin Endocrinol (Oxf) 1997; 46: 719-25.

13. Burrow GN, Fisher DA, Larsen PR. Maternal and fetal thyroid function. N Engl J Med 1994; 331: 1072-8.

14. Glinoer D. The thyropid in pregnancy: a European perspective. Thyroid Today $1995 ; 18: 1-11$.
15. Mestman JH, Goodwin TM, Montoro MM. Thyroid disorders of pregnancy. Endocrinol Metab Clin North Am 1995; 24: 41-71.

16. Leung AS, Millar LK, Koonings PP, Montoro M, Mestman JH. Perinatal outcome in hipothyroidism complicating pregnancy. Obstet Gynecol 1988; 72: 108-2.

17. Smyth PPA. Variation in iodine handling during normal pregnancy. Thyroid 1999; 9: 637-42.

18. Cao XY, Jiang XM, Dou ZH, Rakeman MA, Zhang ML, O'Donnell K, Ma T, Amette K, Delong N, Delong GR. Timing of vulnerability of the brain to iodine deficiency in endemic cretinism. N Engl J Med 1994; 331: 1739-44.

19. Klein RZ, Haddow JE, Faix JD, Brown RS, Hermos RJ, Pulkkinen A, Mitchell ML. Prevalence of thyroid deficiency in pregnant women. Clin Endocrinol 1991; 35: 41-6.

20. Fukushi M, Honma K, Fujita K. Maternal thyroid deficiency during pregnancy and subsequent neuropsychological development of the child [letter]. N Engl Med 1999; 341: 2016.

21. Montoro MN. Management of hypothyroidism during pregnancy. Clin Obstet Gynecol 1997; 40: 65-80.

22. Leung AS, Milar LK, Koonings PP, Montoro MM, Mestman JH. Perinatal outcome in hypothyroid pregnancies. Obstet Gynecol 1993; 81: 349-53.

23. Davis LE, Leveno KJ, Cunningham FG. Hypothyroidism complicating pregnancy. Obstet Gynecol 1988; 72 : 108-12.

24. Wasserstrum N, Anania CA. Perinatal consequences of maternal hypothyroidism in early pregnancy and inadequate replacement. Clin Endocrinol (Oxf) 1995; 42: 353-8.

25. Vaquero E, Lazzarin N, De Carolis C, Valensise H, Moretti C, Ramanini C. Mild thyroid abnormalities and recurrent spontaneous abortion: diagnostic and therapeutical approach. Am J Reprod Immunol 2000; 43: 204-8.

26. Glinoer D. Potential repercussions for the progeny of maternal hypothyroxinemia during pregnancy. Thyroid 2000; 10: 59-62.

27. Lazarus JH. Clinical manifestations of postpartum thyroid disease. Thyroid 1999; 9: 685-9

28. Glinoer D. Management of hypo-and hyperthyroidism during pregnancy. Growth Horm IGF Res 2003; 13: S45-S54. 
29. DeGroot LJ, Larsen PR, Hennesmann G. Thyroid dysfunction in the pregnant patient. In: Glinoer D. Thyroid and its diseases. 6th ed. New York: Churchill Livingstone; 1996. p. 519-40.

30. Mandel SJ, Larsen PR, Seely EW, Brent GA. Increased need for thyroxine during pregnancy in womem with primary hypothyroidism. N Engl J Med 1990; 323: 91-5.

31. Kaplan MM. Monitoring thyroxine treatment during pregnancy. Thyroid 1992; 2: 147-52.

32. Goldman JM. Postopartum thyroid dysfunction. Arch Intern Med 1986; 146: 1296-9.

33. Barca MF, Knobel M, Tomomori E, Cardia MS, Medeiros Neto G. Prevalence and characteristics of postpartum thyroid dysfunction in São Paulo, Brazil. Clin Endocrinol (Oxf) 2000; 53: 21-31.
34. Lucas A, Pizarro E, Granada ML, Salinas I, Foz M, Sanmarti A. Postpartum thyroiditis: epidemiology and clinical evolution in a nonselected population. Thyroid 2000; 10: 71-7.

35. Lazarus JH, Parkes AB, Hall R, Adams H, Premawardhana. influence of postpartum thyroiditis and antiTPO antibodies on symptomatology in the postpartum period. J. Endocrinol. 1993; 137: 208-10.

36. Roti E, Emerson CH. Postpartum thyroiditis. J Clin Endocrinol 1992; 74: 3-5.

37. Lazarus, JH, Parkes AB, Premawardhana LDKE, Harris B Screening for postpartum thyroidits. J Clin Endocrinol Metab 1999; 84: 1-3.

Recebido em 12 de fevereiro de 2004

Versão final apresentada em 12 de maio de 2004

Aprovado em 14 de julho de 2004 